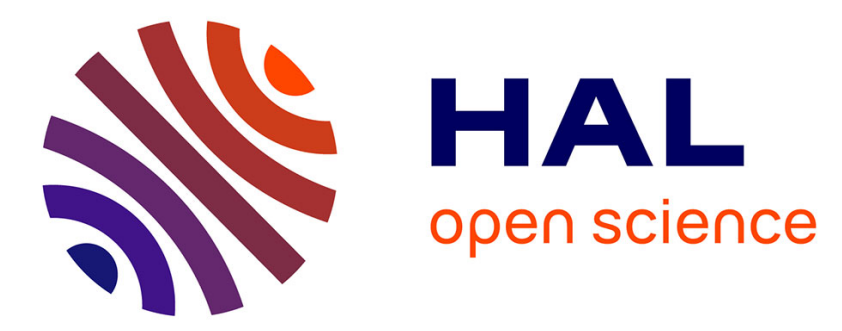

\title{
From Molecular Autoionization to Thermionic Emission.
}

Franck Lepine, Bruno Baguenard, Bruno Concina, Marie-Ange Lebeault, Christian Bordas

\section{To cite this version:}

Franck Lepine, Bruno Baguenard, Bruno Concina, Marie-Ange Lebeault, Christian Bordas. From Molecular Autoionization to Thermionic Emission.. Molecular Physics, 2007, 105 (11-12), pp.16311641. 10.1080/00268970701390198. hal-00513099

\section{HAL Id: hal-00513099 \\ https://hal.science/hal-00513099}

Submitted on 1 Sep 2010

HAL is a multi-disciplinary open access archive for the deposit and dissemination of scientific research documents, whether they are published or not. The documents may come from teaching and research institutions in France or abroad, or from public or private research centers.
L'archive ouverte pluridisciplinaire HAL, est destinée au dépôt et à la diffusion de documents scientifiques de niveau recherche, publiés ou non, émanant des établissements d'enseignement et de recherche français ou étrangers, des laboratoires publics ou privés. 


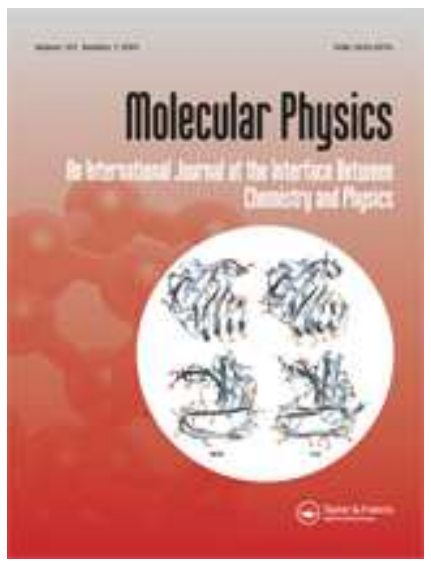

\section{From Molecular Autoionization to Thermionic Emission.}

\begin{tabular}{|c|c|}
\hline Journal: & Molecular Physics \\
\hline Manuscript ID: & TMPH-2007-0035.R1 \\
\hline Manuscript Type: & Full Paper \\
\hline $\begin{array}{r}\text { Date Submitted by the } \\
\text { Author: }\end{array}$ & 01-Apr-2007 \\
\hline Complete List of Authors: & $\begin{array}{l}\text { LEPINE, FRANCK; Universite Lyon 1, LASIM } \\
\text { BAGUENARD, BRUNO; Universite Lyon 1, LASIM } \\
\text { CONCINA, BRUNO; Universite Lyon 1, LASIM } \\
\text { LEBEAULT, MARIE-ANGE; Universite Lyon 1, LASIM } \\
\text { BORDAS, CHRISTIAN; Universite Lyon 1, LASIM }\end{array}$ \\
\hline Keywords: & Autoionization, Rydberg states, Thermionic emission, Clusters \\
\hline
\end{tabular}

\section{s ScholarONE" \\ Manuscript Central}




\title{
From Molecular Autoionization to Thermionic Emission.
}

\author{
F. Lépine, B. Baguenard, B. Concina, M.A. Lebeault, and C. Bordas. \\ Université Lyon 1; CNRS; LASIM UMR 5579,
} 43 Bvd. du 11 novembre 1918, 69622 Villeurbanne, France.

\begin{abstract}
Autoionization of Rydberg states and delayed ionization are two decay processes where the excess energy in the molecule or cluster is released by ejecting a free electron. While precise spectroscopic studies of molecular autoionization are commonly performed, allowing a very detailed understanding of the ongoing processes, the study of delayed ionization in clusters is by far less well known and its description is noticeably less precise. Indeed, in complex systems such as clusters, only a statistical approach is found to be relevant as describing the decay dynamics of the system. Beyond these apparent profound differences we show that both phenomena are actually two facets of the same general phenomenon and that delayed ionization, or thermionic emission in clusters, are analogous to molecular autoionization. The transition from autoionization of Rydberg states to delayed ionization, depicted as a transition from a process entirely described within the framework of quantum mechanics to a process described only in the framework of statistical mechanics may further be foreseen as a prototype of the transition from quantum to classical dynamics.
\end{abstract}




\section{Introduction.}

The study of small gas-phase clusters, and more specifically the study of the decay dynamics of very excited clusters, is a very active field of research [1]. Owing to the combination of relatively simple description and wealth of phenomena that they exhibit, small clusters may indeed be considered as prototypes of finite-size systems that share common properties of small molecules and bulk matter. Despite being formed of a very limited number of atoms, properties of clusters may generally not be described by a full microscopic approach. On the contrary, a statistical description [2] is very often more powerful to describe quantitatively cluster dynamics. While small clusters are most of the time studied as a scale model of bulk matter, all decay processes that they exhibit have their counterpart in small diatomic molecules. In this contribution we will focus on the decay channels involving ejection of a free electron. More precisely, we wish to emphasize the analogy between molecular autoionization [3] (or autodetachment) and delayed ionization [4] (also depicted as thermionic emission) in clusters.

When a diatomic molecule $\mathrm{AB}$ is brought in a Rydberg states lying in the continuum of the ionized molecule $\mathrm{AB}+$, plus an electron, the decay of the very excited neutral molecule $\mathrm{AB}^{* *}$ is governed by three main mechanisms:

$$
\mathrm{AB}^{* *} \longrightarrow \begin{array}{lll}
\mathrm{AB}^{+}+\mathrm{e}^{-} & \begin{array}{l}
\text { autoionization } \\
\mathrm{A}+\mathrm{B}^{*} \\
\mathrm{AB}+\mathrm{h} v
\end{array} & \begin{array}{l}
\text { dissociation } \\
\text { radiative decay }
\end{array} \\
\text { (c) }
\end{array}
$$

For excited clusters, despite their larger number of degrees of freedom, all channels can be classified according to the nature of the ejected particle: electron, heavy fragment, of photon.

$$
\mathrm{X}_{\mathrm{n}}^{* *} \longrightarrow \begin{array}{ll}
\mathrm{X}^{+}+\mathrm{e}^{-} & \begin{array}{l}
\text { delayed ionization } \\
\mathrm{X}_{\mathrm{m}}+\mathrm{X}_{\mathrm{n}-\mathrm{m}}^{*} \\
\mathrm{X}_{\mathrm{n}}+\mathrm{h} v
\end{array} \\
\text { fragmentation } & \text { (b) } \\
\text { radiative decay } & \text { (c) }
\end{array}
$$


Note that the term $X_{n}$ has been used for sake of simplicity, the same description holds for heteronuclear systems.

The analogy between autoionization and delayed ionization is obvious from eq. (1) and (2). Both mechanisms are simply the process by which an excited molecular species releases (a fraction of) its energy by ejecting a free electron. In both cases the fundamental motivation for studying these processes answers the same very fundamental question: how do systems excited close to their ionization, or detachment, limit behave? There is however a number of differences between processes (1) and (2). The first one is of experimental, or practical, nature. Though autoionization generally proceeds via the excitation of a well defined autoionizing resonance, a quasi discrete state with more or less well defined quantum numbers, delayed ionization of an excited clusters follow an excitation step that is in principle non selective and that does not allow to define properly the details of the excited states (quantum numbers). In complex systems where the density of states (DOS) is extremely high the excitation of a coherent superposition of quantum states cannot be avoided. This complexity prevents a simple approach assuming the population of a single quantum state, but it makes a classical description of the dynamics much relevant. The second difference regards the theoretical description of both processes. While a microscopic approach (perturbation theory [5], or more specifically Multichannel Quantum Defect Theory MQDT [6-8]) is suitable for the treatment of the autoionization of diatomic molecules, no such microscopic approach may be followed to describe decay dynamics of clusters. Rather, a statistical approach [2, 9-11] is in most cases the only performing tool that can be used to interpret the experimental observations. Although, these differences are not of essential nature and seem to appear only due to our inability to treat in details complex systems such as clusters, they imply a more fundamental difference as they underlie the quantum to classical transition. There is indeed a point where technical limitations such as experimental resolution or computational capacities are not the only obstacles preventing a full quantum description of clusters.

In order to illustrate the similarities between molecular autoionization and delayed ionization in clusters we will present in this manuscript two examples of results obtained in our group over the past years that summarize rather generally these processes. In section 2 we present molecular 
autoionization with the example of (vibrational) autoionization in $\mathrm{Na}_{2}$ Rydberg states [12, 13]. The structure and dynamics of molecular Rydberg series is best described in the framework of MQDT [6-8, 14-16]. However, we will limit the theoretical description to a more standard perturbative approach [5] that has the great advantage to allow a more natural extension to larger systems like clusters. Beside, we will also show in this section that, even for simple diatomic system, the dynamics may be more complex than expected and its complete description may require the introduction of internal dynamics that makes simple molecules even closer to complex systems. In section 3 we will introduce rather briefly the statistical description of delayed ionization in clusters [4] in the framework of the detailed balance theory [9, 17-19]. We will underline the formal equivalence between this approach and the perturbative approach of autoionization. Delayed ionization will be illustrated by the example of $C_{60}$ [19-21]. In that case the (multiphoton) excitation process itself contributes to the statistical nature of the whole process. Finally, section 4 will be devoted to delayed detachment which has been extensively studied in the literature [22-24].

\section{Molecular autoionization.}

\subsection{Simple description of autoionization.}

The detailed mechanisms of autoionization have been extensively studied in the past, in a perturbative approach first [5], and in the framework of Multichannel Quantum Defect Theory (MQDT) subsequently [6-8]. MQDT takes advantage of the asymptotic form of Coulomb function and matching at short distances and allows a non-perturbative approach more powerful than the standard perturbative approach. As far as molecular autoionization is concerned, a unified treatment has been developed by C. Jungen [7, 8, 14-16] that allows a global description of all kinds of molecular autoionization and dissociation processes. MQDT has also been extensively applied to the case of $\mathrm{Na}_{2}$ Rydberg states [13] presented below as an illustration. However, in the present article, a summary of the perturbative approach will be used instead since it allows a more qualitative comparison with delayed ionization in clusters. 
Autoionizing states are quasi-discrete states lying above the lowest ionization threshold, members of Rydberg series converging to an excited level of the ion. According to the nature of the excitation of the ion various kinds of autoionization processes are found. Autoionization involves a non-radiative exchange of energy between the core and the outer electron. The origin of the core energy transferred to the electron may be rotational, vibrational, spin-orbit, or electronic depending on which processes are energetically possible. In a typical molecule like $\mathrm{Na}_{2}$, the most common process is vibrational autoionization. Spin-orbit as well as rotational energies are indeed rather small and corresponding autoionization processes are confined to narrow spectral regions. On the other hand, electronic autoionization is found only in doubly excited states [25]. Vibrational autoionization is schematized in figure 1. If a Rydberg state of an $\mathrm{AB}$ molecule belonging to a series converging towards a $\mathrm{v} \neq 0$ level of the $\mathrm{AB}^{+}$ion lies above the $\mathrm{v}=0$ level of the ion, autoionization occurs by a breakdown of the Born-Oppenheimer approximation. The electronic wavefunction for the quasi-discrete state of the excited neutral is coupled to the one of the ion plus free electron system via the vibronic kinetic energy operator $\hat{\mathrm{T}}_{\mathrm{vib}}$. Autoionization occurs when one $(\Delta \mathrm{v}=1)$ or several $(\Delta \mathrm{v}>1)$ quanta of vibration energy in the neutral are converted to kinetic energy of the outer electron, resulting in its ejection.

Generally the quantum transition amplitude between an initial (i) and a final (f) state may be written according to first order perturbation theory:

$$
\mathrm{a}_{\mathrm{if}}=\frac{2 \pi}{\mathrm{h}}\left|\left\langle\mathrm{i}\left|\mathrm{T}_{\mathrm{if}}\right| \mathrm{f}\right\rangle\right|^{2}
$$

In section 2.2 below the qualitative differences between $\Delta v=1$ and $\Delta v>1$ processes will be illustrated in practical terms. The autoionizing initial Rydberg states is specified by 1 (including all other quantum numbers), $\mathrm{n}$ (principal quantum number), and v (vibrational quantum number), and the final state by $2, \mathrm{v}^{+}$(vibrational quantum number of the resulting ion), and $\varepsilon$ (kinetic energy of the ejected electron).

The corresponding partial autoionization width of state $\left|\Psi_{1, \mathrm{n}, \mathrm{v}}\right\rangle$ with respect to the continuum $\left|\Psi_{2, \varepsilon, \mathrm{v}^{+}}\right\rangle$may be expressed by [5]: 


$$
\Gamma_{1, \mathrm{n}, \mathrm{v} ; 2, \varepsilon, \mathrm{v}^{+}}=2 \pi\left|\left\langle\Psi_{1, \mathrm{n}, \mathrm{v}}\left|\mathrm{T}_{\mathrm{if}}\right| \Psi_{2, \varepsilon, \mathrm{v}^{+}}\right\rangle\right|^{2}
$$

As a first approximation the interaction is factored into electronic and nuclear parts and the matrix element in eq.(4) reduces to:

$$
\left\langle\Psi_{1, \mathrm{n}, \mathrm{v}}\left|\mathrm{T}_{\mathrm{if}}\right| \Psi_{2, \varepsilon, \mathrm{v}^{+}}\right\rangle=\left\langle\Phi_{1, \mathrm{n}}\left|\mathrm{T}_{\mathrm{if}}\right| \Phi_{2, \varepsilon}\right\rangle\left\langle\mathrm{v} \mid \mathrm{v}^{+}\right\rangle
$$

Note that the electronic part of the continuum wavefunction $\Phi_{2, \varepsilon}$ is energy-normalized. Owing to the specific character of Rydberg states, the electronic part of the matrix element may be approximated by:

$$
\left\langle\Phi_{1, \mathrm{n}}\left|\mathrm{T}_{\mathrm{if}}\right| \Phi_{2, \varepsilon}\right\rangle=\frac{2 \mathfrak{R}}{\mathrm{n}^{3}} \mathrm{I}
$$

Where $\mathfrak{R}$ is the Rydberg constant, and I is a dimensionless parameter almost independent of $\mathrm{n}$ characterizing the interaction. In the case of vibrational autoionization, the matrix elements appearing in the above equations may be expressed as a function of the R-dependent quantum defect $\mu_{\mathrm{n} \ell \lambda}(\mathrm{R})$ defined as follows [26]:

$$
\mathrm{U}_{\mathrm{n} \ell \lambda}(\mathrm{R})=\mathrm{U}^{+}(\mathrm{R})-\frac{\mathfrak{R}}{\left(\mathrm{n}-\mu_{\mathrm{n} \ell \lambda}(\mathrm{R})\right)^{2}}
$$

Where $\mathrm{R}$ is the internuclear distance, $\mathrm{U}_{\mathrm{n} \ell \lambda}(\mathrm{R})$ is the potential energy curve of the Rydberg state, and $\mathrm{U}^{+}(\mathrm{R})$ the potential energy curve of the ion. $\mu_{\mathrm{n} \ell \lambda}(\mathrm{R})$ is a slowly varying function of $\mathrm{R}$, almost independent of $n$. Keeping only the first order term in the Taylor development of $\mu_{n \ell \lambda}(R)$ and in the harmonic approximation, the only nonzero matrix elements $\left\langle\Psi_{1, \mathrm{n}, \mathrm{v}}\left|\mathrm{T}_{\mathrm{if}}\right| \Psi_{2, \varepsilon, \mathrm{v}^{+}}\right\rangle$are those corresponding to $\Delta \mathrm{v}=\mathrm{v}^{+}-\mathrm{v}=-1$. This approximate selection rule is known as the propensity rule 


\subsection{Rovibrational autoionization in $\mathrm{Na}_{2}$.}

Owing to their relatively low ionization potentials and to their simple electronic structure, alkali metal diatomic molecules are often used as model systems for molecular Rydberg states. The sodium dimer for instance has been extensively studied in the past decades [12, 13, 28, 29] and will be used to illustrate our comments. $\mathrm{Na}_{2}$ is produced as a molecular beam in a thermal oven. The optical excitation is achieved using tunable pulsed lasers ( $\mathrm{Nd}$ :Yag pumped dye lasers, $10 \mathrm{~ns}$ duration, $0.2 \mathrm{~cm}^{-1}$ spectral linewidth) in an interaction region where a small electric field (from 0.3 to a few hundreds $\mathrm{V} / \mathrm{cm}$ according to the experimental purpose) is applied. The external electric field may be static or pulsed in order to excite field-free states. It is used both to collect the charged particles ejected during the ionization process (electrons or ions) and to study its effect on very 
excited states. We use a selective optical-optical double resonance excitation process in order to excite single Rydberg series avoiding spectral congestion. The first tunable dye laser $h v_{1}$ is tuned to a well-defined rovibrational transition between the ground $\mathrm{X}^{1} \Sigma_{\mathrm{g}}^{+}$and first excited $\mathrm{A}^{1} \Sigma_{\mathrm{u}}^{+}$state. This allows selective excitation of the $\mathrm{Na}_{2}$ molecule in a single (v', J') level of the intermediate $\mathrm{A}^{1} \Sigma_{\mathrm{u}}^{+}$state. From this intermediate level the second dye laser $\mathrm{h} v_{2}$ is scanned in the vicinity of the ionization threshold to excite gerade Rydberg states with $\mathrm{J}=\mathrm{J}^{\prime}$ or $\mathrm{J}^{\prime} \pm 1$. Owing to the Rydberg character of the A state, the $\mathrm{v}=\mathrm{v}$ ' Rydberg series are preferentially excited. This allows an additional clarification of the Rydberg spectra. Typical spectra are found e.g. in ref. [12] that describes one of the most striking aspects of those Rydberg states: namely the stroboscopic effect between the electronic and nuclear motion that occurs when the classical frequency of the electronic motion $\omega_{\mathrm{el}} \approx 2 \mathfrak{R} / \mathrm{n}^{3}$ is commensurable with the classical frequency of the nuclear rotational period $\omega_{\mathrm{N}} \approx 2 \mathrm{~B}(\mathrm{~J}+1 / 2)$ (with $\mathrm{B}$ the rotational constant). This stroboscopic effect is visualized by a drastic simplification of the spectrum whenever $2 \omega_{\mathrm{N}}=\mathrm{k} \omega_{\mathrm{el}}$ (with $\mathrm{k}$ integer).

Figure 2 presents the typical behavior of $\mathrm{Na}_{2}$ Rydberg states with respect to autoionization $[13,28]$. Both spectra presented in this figure have been recorded with the intermediate level $\left(v^{\prime}=2\right.$, $\mathrm{J}^{\prime}=6$ ). Only the $\mathrm{v}=2$ Rydberg series is visible in these spectra. The upper spectrum has been recorded using an integration time window of $200 \mathrm{~ns}$ (i.e. all electrons ejected during the first $200 \mathrm{~ns}$ after laser excitation are detected), while the lower spectrum has been recorded with a shorter integration window of $10 \mathrm{~ns}$. The comparison of both spectra gives therefore a direct and qualitative view of the excited states lifetime. This method allows a more global view of the dynamics than the examination of the lifetime of individual lines. Indeed, the ratio of the relative intensities of similar lines in the two spectra is directly connected to the lifetime of the excited states. Figure 2 exhibits clearly that the lifetime of autoionizing Rydberg states is typically a few nanoseconds just above the $\Delta v=1$ autoionization threshold, while it increases to several tens of nanoseconds as $\mathrm{n}$ increases just below the $\mathrm{v}^{+}=2$ ionization limit. The patterns observed for $\Delta \mathrm{v}=2$ autoionizing states show that lifetime is significantly larger in that case (always larger than 10$20 \mathrm{~ns}$ ), but show at the same time that the $\Delta \mathrm{v}=1$ rule is only a propensity rule. Below the direct $(\Delta \mathrm{v}=0)$ ionization threshold $\left(\mathrm{v}^{+}=2\right)$ and below the $\Delta \mathrm{v}=1$ ionization threshold $\left(\mathrm{v}^{+}=1\right)$ the long lifetimes corresponding to vanishing widths are clearly evidenced on the lower spectrum. Note that 
the various ionization thresholds are lowered by the external electric field $(1.5 \mathrm{~V} / \mathrm{cm})$. The average autoionization lifetime may be estimated from such spectra. Figure 3 presents such an estimation in the case of Rydberg states excited via the $\left(v^{\prime}=1, J^{\prime}=6\right)$ intermediate level for different values of the electric field. Experimental data recorded at different values of the external electric field (F=2, 5 and $20 \mathrm{~V} / \mathrm{cm}$ ) are compared with theoretical spectrally averaged lifetimes calculated in the framework of MQDT [28]. Figure 3 shows clearly the global increase of autoionization lifetime as a function of the principal quantum number $n$, as well as the increasing of the lifetime as a function of the electric field in a given spectral region. This field-hindered vibrational autoionization is qualitatively explained by the dilution of the coupling between quasi bound Rydberg states and the ionization continuum. Indeed, the electric field couples the low- $\ell$ penetrating Rydberg states with the high- $\ell$ states that are only weakly coupled to the ionization continuum owing to their vanishing wavefunction amplitude in the core region.

The various experimental results presented in this section allow drawing a few rather general conclusions regarding the nature of vibrational autoionization in diatomic molecules. First, the autoionization lifetime is directly dependent on the precise nature of the excited state and it depends critically on the nature and quantity of energy transferred from the nuclear to the electronic degree of freedom, as well as on the effective principal quantum number. A perturbative approach, or even better a full MQDT description, allows estimating rather quantitatively autoionization lifetimes of the various Rydberg states. From this point of view it is clear that the autoionization lifetime does not depend simply on the electron kinetic energy. However it depends very predictably upon the nature of the excited state. Second, it is also obvious from these results that a rather weak perturbation may influence drastically the effective dynamics of autoionization.

In addition, a simple diatomic molecule may exhibit a non trivial dynamics and mimic the dynamics of a complex system. This evolution towards complexity is discussed below.

\subsection{Non-exponential autoionization decays and evolution towards complexity.}

For a single isolated autoionizing state, a total autoionization rate may be defined. The autoionization lifetime is inversely proportional to this total decay rate. In principle the decay of such state is expected to be exponential in time. However, there is a number of situations where this 
simple behavior is no longer observed as it is illustrated in figure 4 in the case of a particular Rydberg state $\left(\mathrm{n}=17 \mathrm{~d} \Pi^{-}, \mathrm{v}=4, \mathrm{~J}=6\right)$ [29]. In the presence of a low electric field $(\mathrm{F}=1 \mathrm{~V} / \mathrm{cm})$, the decay of this Rydberg state is roughly exponential. When the electric field is increased to only $2.4 \mathrm{~V} / \mathrm{cm}$ the decay dynamics of this line is qualitatively different and the photoelectron signal increases with a typical rise time of $60 \mathrm{~ns}$ (much larger than the laser pulse duration $\approx 10 \mathrm{~ns}$ ) and a decay time of about 95 ns. A simple model [29], assuming the excitation of a single autoionizing state $|a\rangle$ indirectly coupled to the ionization continuum via a dark state $|b\rangle$ was used to simulate this ionization signal. In the context of ZEKE spectroscopy [30] such non-exponential decays have been observed subsequently in high-n Rydberg states. Multichannel effective Hamiltonians including several doorway states (for excitation and decay) and pure escape states (for decay) were introduced [31], generalizing our simple approach. The extreme sensitivity to the external electric field makes the detailed interpretation of this phenomenon extremely difficult. However, we learned from this observation that, in some cases, molecular Rydberg states behave like complex excited systems: by tuning the strengths of the internal couplings with the external electric field, the dynamics of decay may change drastically and present a complex behavior resulting from the balance between internal couplings and couplings to the continuum. This represents a step forward complexity in the sense that it introduces the notion of internal energy relaxation and a possible statistical description.

\section{Delayed ionization of clusters.}

\subsection{Delayed ionization as thermionic emission.}

When the size of the molecule increases a more complex ionization dynamics is expected owing to the influence of the increasing number of degrees of freedom. Let us consider a photoexcited molecule with an internal energy exceeding the lowest ionization threshold. In a simple picture, the excitation process can produce a state that is either a state of the continuum or a quasi-bound excited state. In the later case, this state may be directly coupled to the continuum but also be influenced by another perturbation (electric field, other degrees of freedom). Simple DOS 


\title{
3.2. Formal link between autoionization and thermionic emission in neutral species.
}

One of the major ingredients justifying the validity of the statistical description of the decay process is the high DOS of the molecule in the energy range under consideration. We consider an energetic state of the system where many quantum states are accessible in the range $\{\mathrm{E}, \mathrm{E}+\Delta \mathrm{E}\}$. A

high DOS ensures that the number of states involved remains large even if the width $\Delta \mathrm{E}$ of the

\begin{abstract}
arguments show that the coupling to other quasi-bound states is in general larger than the direct coupling to the ionization continuum. Due to the couplings between the various degrees of freedom, the excitation is no longer localized and the energy is distributed in the molecule, with a constant exchange of energy between electrons and nuclei.
\end{abstract}

The quantity of energy accumulated on a given degree of freedom fluctuates owing to the various couplings and these fluctuations, corresponding to an equipartition of the energy over all accessible degrees of freedom, may be described statistically. If at some point the energy accumulated on a given vibrational mode is higher than the binding energy of the atom in this mode, dissociation may occur. This description in terms of statistical distribution of the internal energy constitutes the background of the so-called RRK [10] theory that has been successfully applied to the description of unimolecular reactions [2].

As far as ionization is concerned, electron delayed emission from a highly excited system may be described in a very similar manner. When delayed ionization follows an equipartition of the internal energy over all degrees of freedom, it may be described as a statistical process and is denoted as thermionic emission [11, 17-19]. In order to be efficient as compared to direct ionization, internal energy redistribution must occur rapidly after the initial excitation. From the microscopic point of view, the electron can gain enough energy from the other electrons or from the nuclei. In the later case, the "kick" received by the electron from the nuclei corresponds strictly to the vibrational autoionization process discussed in the previous section. In the following, we discuss how molecular autoionization (of quantum nature) and statistical (classical) delayed electron emission can be formally linked. This transition corresponds to the loss of the quantum character of the ionization process: a specific example of the very general problem of the transition between microscopic quantum process and classical / statistical behavior. 
energy range vanishes. In order to describe the emission process, the corresponding rate has to take into account the population of final states. According to Fermi's golden rule [32] the average emission rate for the process leading to the ejection of an electron with a kinetic energy $\varepsilon$ from an initial state $|i\rangle$ at the total energy $E$ in a continuum state $|f\rangle$ may be expressed as follows:

$$
\mathrm{k}_{\mathrm{em}}(\mathrm{E}, \varepsilon)=\frac{2 \pi}{\mathrm{h}}\left|\left\langle\mathrm{i}\left|\mathrm{T}_{\mathrm{if}}\right| \mathrm{f}\right\rangle\right|^{2} \rho_{\mathrm{f}}\left(\mathrm{E}-\mathrm{E}_{\mathrm{b}}, \varepsilon\right)
$$

Where $\rho_{\mathrm{f}}(\mathrm{E}, \varepsilon)$ is the final DOS and $\mathrm{E}_{\mathrm{b}}$ the electron binding energy. The rate may be expressed as the product of the DOS of the free ejected electron and the DOS of the remaining system. The explicit calculation of this rate requires the knowledge of the detailed vibronic transitions and therefore information on both nuclear and electronic ground and excited states. This is hardly possible for a molecule or a cluster containing more than few tens of atoms. In order to be able to describe the electron emission process it is suitable to make use of average quantities. A macroscopic approach is often used when delayed electronic emission is considered. It is based on the "detailed balance" theory [9] and allows calculating the emission rate from the known rate of the reverse process, namely electron capture.

The reverse average rate of the emission process is expressed as:

$$
\mathrm{k}_{\mathrm{rev}}(\mathrm{E}, \varepsilon)=\frac{2 \pi}{\mathrm{h}}\left|\left\langle\mathrm{f}\left|\mathrm{T}_{\mathrm{fi}}\right| \mathrm{i}\right\rangle\right|^{2} \rho_{\mathrm{i}}(\mathrm{E})
$$

Where $\rho_{\mathrm{i}}(\mathrm{E})$ is the DOS in the initial step.

Using the definition of the reverse process cross section, this rate may also be expressed as follows:

$$
\mathrm{k}_{\mathrm{rev}}(\mathrm{E}, \varepsilon)=\frac{\mathrm{v}}{\mathrm{V}} \sigma(\varepsilon, \mathrm{E})
$$


Where $\mathrm{v}$ is the relative velocity of the fragments, $\mathrm{V}$ an arbitrary volume and $\sigma(\varepsilon, \mathrm{E})$ the electron capture cross-section. Note that eqs. (10) and (11) are different in the sense that the former relies on quantum transitions whereas the later makes use of average quantities.

Using the definition of the transition amplitude given by eq. (3) and according to the microreversibility principle:

$$
\mathrm{a}_{\mathrm{ij}}=\mathrm{a}_{\mathrm{ji}}
$$

We can combine eqs. (10), (11), and (12) in order to express the rate for the emission of a particle with kinetic energy $\varepsilon$ from a molecule of total internal energy $E$ [9, 17-19]. We obtain:

$$
\mathrm{k}_{\mathrm{em}}(\varepsilon, \mathrm{E})=\frac{2 \mathrm{~m}}{\pi^{2} \hbar^{3}} \sigma(\varepsilon, \mathrm{E}) \varepsilon \frac{\rho_{\mathrm{d}}\left(\mathrm{E}-\mathrm{E}_{\mathrm{b}}-\varepsilon\right)}{\rho_{\mathrm{i}}(\mathrm{E})}
$$

This equation may also be written as follows:

$$
\mathrm{k}_{\mathrm{em}} \rho_{\mathrm{i}}=\mathrm{k}_{\mathrm{rev}} \rho_{\mathrm{f}}
$$

This expression is known as the Weisskopf relation [9], macroscopic counterpart of the microscopic detailed balance theory.

A step further consists in introducing the microcanonical temperature [17] of the molecule or cluster via the ratio of DOS using its definition:

$$
\frac{1}{\mathrm{k}_{\mathrm{B}} \mathrm{T}}=\frac{\partial \ln \rho(\mathrm{E})}{\partial \mathrm{E}}
$$

At this point it is assumed that the DOS corresponds to the density of vibrational states and the calculated temperature is the vibrational microcanonical temperature. The cross-section in eq. 
(11) may be explicitly estimated via a classical approach. Combination of eqs. (13) and (15) allows deriving the electron kinetic energy spectrum experimentally accessible:

$$
\mathrm{P}(\varepsilon, \mathrm{E}) \propto\left(\varepsilon+\mathrm{C} \varepsilon^{\gamma}\right) \exp \left(-\frac{\varepsilon}{\mathrm{k}_{\mathrm{B}} \mathrm{T}_{\mathrm{d}}(\mathrm{E})}\right)
$$

Where $T_{d}$ is the microcanonical temperature of the system after the electron loss. The preexponential factor consists in two terms. The first one $(\varepsilon)$ is given by the contribution of the hard sphere part of the electron capture cross section, the second one depends explicitly on the interaction between the electron and the remaining system $(\gamma=0$ in case of delayed ionization, $\gamma=1 / 2$ in case of delayed detachment).

Note that the kinetic energy spectrum is structureless and entirely determined by only two parameters: the temperature $\mathrm{T}_{\mathrm{d}}$ related to the DOS, and the exponent $\gamma$ given by the cross-section. The total decay rate is simply obtained by integrating eq. (13) over $\varepsilon$. This rate increases with the internal energy (i.e. with temperature). This behavior is a signature of the statistical character of the process and is generally not observed in the autoionization of small molecules Rydberg states.

During the last few years, we have developed an experimental set-up that allows the measurement of the kinetic energy spectrum of photoelectrons ejected in delayed ionization of size selected clusters, at a given time delay after the excitation process. This set-up is based on a velocity map imaging spectrometer [33] combined with a time-resolved position sensitive detector [34]. In the following we discuss how this technique has been applied to neutral and charged clusters in order to study delayed emission.

\subsection{Time resolved photoelectron imaging: $\mathbf{C}_{60}$.}

Our time resolved photoelectron imaging technique takes advantage of the fact that the emission rate describing statistical emission has a very fast evolution with the internal energy. This means that two systems having a close internal energy have a very different lifetime $\tau$. As a consequence the measurement of the electron kinetic energy spectrum at a given time delay $\tau$ corresponds to the detection of electrons emitted from systems having a rather well defined internal 
energy E. A microcanonical temperature $T_{d}(E)$ may be associated to this internal energy and may be measured by fitting to the kinetic energy spectrum. In the case of a thermionic process, the temperature $T_{d}(E)$ measured at the time delay $\tau$ is determined by the total emission rate according to the approximate relation $\mathrm{K}(\mathrm{E})=1 / \tau$.

As far as delayed ionization of finite-size systems is concerned the fullerene $\mathrm{C}_{60}$ is undoubtedly the molecule or cluster that has attracted the largest attention $[1,35,36]$. Indeed $\mathrm{C}_{60}$, owing to its high symmetry and large number of degrees of freedom, has often be used as the prototype of small systems for studying delayed emission processes. It is particularly interesting because the electron emission process is in competition with the dissociation (emission of $\mathrm{C}_{2}$ ) process that is actually the major decay channel [37]. In other words the total decay rate is almost entirely determined by the dissociation rate. For this system, eq. (16) may be explicitly written with $\mathrm{C}=4.1 \mathrm{eV}$ and $\gamma=0$. Experimental results have been published previously [19, 20]. A typical example of a delayed photoelectron spectrum recorded in multiphoton ionization of $\mathrm{C}_{60}$ (ionization laser: $\lambda=355 \mathrm{~nm}$, pulse duration $=10 \mathrm{~ns}$ ) is presented in figure 5 together with the result of the fit according to eq. (16) where only the temperature $T_{d}$ of the residual ion is a free parameter. Figure 5 shows the very good agreement with the statistical approach. Indeed, the photoelectron spectrum may be used as a thermometer to determine the microcanonical temperature $T_{d}$ of the final product. The fitted value of this temperature may in turn be compared with the statistical model. In that particular case, the electron spectrum is used to test the values of the dissociation rate proposed in the literature. Assuming $\mathrm{K}=10^{21} \exp \left(-10.2 / \mathrm{k}_{\mathrm{B}} \mathrm{T}_{\mathrm{e}}\right)$, a very good agreement is found between experiment $\mathrm{T}_{\mathrm{d}}^{\mathrm{exp}}=3100 \pm 100 \mathrm{~K}$ and theory $\mathrm{T}_{\mathrm{d}}^{\mathrm{th}}=3180 \mathrm{~K}$ at delay $\tau=1 \mu \mathrm{s}$.

Note that the spectrum has no structure as expected from the statistical approach. Measurements performed at different wavelength and intensity [19, 20] show no significant difference with the one presented here, in agreement with the non selective character of the statistical behavior. The very good agreement with Weisskopf theory [9, 19] is not extremely surprising regarding the size of the system due to the very high density of vibrational states involved in the process. 


\subsection{Delayed emission in anions: autodetachment and thermionic emission.}

Delayed ionization of neutral clusters may be viewed as a statistical autoionization process where the energy is randomly accumulated on a specific vibrational mode coupled to continuum states of the ion+electron system. Anion clusters, despite their different nature, represent interesting and widely studied species [22-24]. In particular, during the past years, we have investigated delayed electron emission of anion carbon clusters [38]. While, strictly speaking, it is no longer an ionization process, delayed electron detachment bears many common features with autoionization and delayed ionization. Simons [39] derived a quantum description and discussed propensity rules for autodetachment. This work has to be linked to Berry's results on autoionization [27]. The same link between delayed ionization and delayed detachment in clusters exists. Both processes are depicted as thermionic emission. As far as vibrationally induced electron emission is concerned, neutral and anion systems also share many common features. An additional advantage of negatively charged systems is that mass selection may easily be performed. This is especially useful to investigate the evolution of delayed emission from a small simple system (di- or tri-atomic molecule) to a larger system with many degrees of freedom (like e.g. $\mathrm{C}_{60}^{-}$).

As it has been discussed in the previous section, the connection between autoionization and thermionic emission is intimately related to the transition between discrete and continuous (classical) DOS. This is illustrated on figure 6 where we have calculated the DOS $\rho(E-A E-\varepsilon)$ for the daughter systems for the carbon anion clusters $\mathrm{C}_{6}^{-}$and $\mathrm{C}_{18}^{-}$. DFT calculations have been used to calculate the vibrational modes [40] of the neutral species and a counting procedure has been used to estimate the DOS in the harmonic approximation. In the present calculation we have assumed the internal energy $\mathrm{E}-\mathrm{AE}=1 \mathrm{eV}$. The results are presented on a log-scale plot. The most striking effect is the obvious discrete character of the DOS of the smaller system $\mathrm{C}_{6}^{-}$. Due to the quantized character of the DOS, no temperature can be defined through equation (15). In that example the concept of temperature and of thermionic process in meaningless. For a larger system $\mathrm{C}_{18}^{-}$, the evolution of the DOS with the electron kinetic energy is very smooth and no discrete structure is observed. Moreover, this evolution is roughly exponential (linear in the log plot) as it is expected for a classical system. In that case the concept of temperature is useful and characterizes the amount of internal energy for a given system that is defined through its DOS. This curve is 
compared with a good agreement to the approximation $\exp \left(-\varepsilon / \mathrm{k}_{\mathrm{B}} \mathrm{T}_{\mathrm{d}}\right)$ where $\mathrm{T}_{\mathrm{d}}=576 \mathrm{~K}$ is calculated using eq. (15). For a given internal energy a larger system corresponds to a less structured and more classical DOS.

Measurements of delayed electron kinetic energy spectra were performed on size selected anion carbon clusters, $90 \mathrm{~ns}$ after the excitation process [38]. Comparison between the experimental spectrum and the theoretical prediction is presented in figure 7 for $\mathrm{C}_{18}^{-}$. The determination of the temperature is based on the calculation of the emission rate as explained in the previous section for $\mathrm{C}_{60}$ but in the present case the electron emission process is the major decay channel. A very good agreement is found between theoretical $\left(T_{d}=1500 K\right)$ and experimental results $\left(T_{d}=1400 \pm 100 K\right)$. A similar agreement is also observed for other sizes [38]. In the case of a smaller system like $\mathrm{C}_{6}^{-}$also presented in figure 7. the agreement does not seem to be correct even if the general features of a thermionic spectrum seem to be present. It is obvious that the simple description used for $\mathrm{C}_{18}{ }^{-}$in which the threshold behavior is mainly determined by the exponent $\gamma=1 / 2$ (eq. (16)) will not be able to represent the near exponential shape of this spectrum around $0 \mathrm{eV}$.

Many effects might be responsible for this disagreement. In principle, Weisskopf formalism is able to take into account the so-called size effects and the influence of the cluster shape which might play an important role for a few atoms cluster. This can be done for instance through an accurate determination of the attachment cross section. At the same time, the concept of microcanonical temperature might not be introduced and a direct calculation of the DOS may be used in a basic formulation of Weisskopf theory (eq. (13)). But in any case, the statistical theory will fail to describe a system where the DOS is not large enough since average quantities on which it is based become meaningless. Therefore, even the introduction of refined model based on statistical theory will not be able to describe delayed emission from very small clusters and statistical representation has to be replaced by a detailed quantum approach where autoionization (or autodetachment) will play a major role. 


\section{Conclusion.}

The experimental results presented above, both in the case of vibrational autoionization of diatomic molecules Rydberg states and in the case of delayed ionization or thermionic emission of clusters, illustrate how small systems exhibit increasingly complex dynamical properties as their size increases. These results show also that this evolution is not necessarily smooth as a function of the size of the molecule, owing to the explosive character of the evolution of the density-of-states vs. size.

Although a theoretical description of this transition in a model system is attainable, the detailed experimental characterization of this transition from quantum to classical behavior is more demanding. Among other requirements, the control of the initial internal energy of the clusters is necessary. In order to fulfill this experimental constraint, we are now building a thermalized cluster source in our laboratory. Provided a full control of the initial state of the cluster, measurements on small size-selected species using time resolved imaging spectrometry should allow us to give additional insight into the transition between autoionization and thermionic emission.

\section{Acknowledgements.}

CB warmly acknowledges fruitful discussions with Christian Jungen in the early stages of this work. Contributions of Michel Broyer, Serge Martin, Pierre Labastie, Jacques Chevaleyre, PierreFrançois Brevet, and Bernard Tribollet to the work on $\mathrm{Na}_{2}$ Rydberg states are gratefully acknowledged. The work on clusters has also benefited from enlightening discussions with Klavs Hansen and Florent Calvo. 


\section{References}

[1] Special issue on cluster cooling, Int. J. Mass. Spectrom. 252 (2006) ed. by K. Hansen and C. Bordas.

[2] Unimolecular reactions, a concise introduction, Wendell Forst (Cambridge University Press, 2003).

[3] Rydberg States of Atoms and Molecules, ed. by R.F. Stebbings, F.B. Dunning (Cambridge Univ. Press, Cambridge 1983).

[4] E.E.B. Campbell and R.D. Levine, Annu. Rev. Phys. Chem. 51 (2000) 65.

[5] Perturbations in the Spectra of Diatomic Molecules, H. Lefebvre-Brion, R.W. Field, (Academic Press Inc. London, 1986).

[6] M.J. Seaton, Proc. Phys. Soc. 88 (1966) 801; 88 (1966) 815.

[7] Ch. Greene, C. Jungen, Adv. Atom. Mol. Phys. 21 (1985) 51.

[8] C. Jungen, O. Atabek, J. Chem. Phys. 66 (1977) 5584.

[9] V. Weisskopf, Phys. Rev. 52 (1937) 295.

[10] O.K. Rice, H.C. Ramsperger, J. Amer. Chem. Soc. 49 (1927) 1617; L.S. Kassel, J. Phys. Chem. 35 (1928) 1065; R.A. Marcus, J. Chem. Phys. 20 (1952) 359.

[11] C.E. Klots, J. Phys. Chem. 92 (1988) 5864; J. Chem. Phys. 90 (1989) 4470; J. Chem. Phys. 98 (1993) 1110; J. Chem. Phys. 100 (1994) 1035.

[12] P. Labastie, M.C. Bordas, B. Tribollet, M. Broyer. Phys. Rev. Lett. 52 (1984) 1681.

[13] C. Bordas, P. Labastie, J. Chevaleyre, M. Broyer. Chem. Phys. 129 (1989) 21.

[14] C. Jungen, D. Dill, J. Chem. Phys. 73 (1980) 3338.

[15] M. Raoult, C. Jungen, J. Chem. Phys. 74 (1981) 3388.

[16] C. Jungen, Phys. Rev. Letters 53 (1984) 2394

[17] J.U. Andersen, E. Bonderup, K. Hansen, J. Chem. Phys. 114 (2001) 6518.

[18] J.U. Andersen, E. Bonderup, K. Hansen, J. Phys. B, 35 (2002) R1.

[19] F. Lépine, C. Bordas. Phys. Rev. A 69 (2004) 053201.

[20] F. Lépine, B. Climen, F. Pagliarulo, B. Baguenard, M.A. Lebeault, C. Bordas, M. Hedén. Eur. Phys. J. D 24 (2003) 393.

[21] C. Bordas, B. Baguenard, B. Climen, M.A. Lebeault, F. Lépine, F. Pagliarulo. Eur. Phys. J. D 34 (2005) 151. 
[22] H. Weidele, D. Kreisle, E. Recknagel, G. Schulze Icking-Konert, H. Handschuh, G. Ganteför, W. Eberhardt. Chem. Phys. Letters 237 (1995) 425.

[23] G. Ganteför, W. Eberhardt, H. Weidele, D. Kreisle, E. Recknagel, Phys. Rev. Lett. 77 (1996) 4524.

[24] C.E. Klots, R.N. Compton. Phys. Rev. Letters, 76 (1996) 4092.

[25] C. Bordas, J.L. Vialle, M. Broyer. Phys. Rev. Lett. 64 (1990) 1223.

[26] R.S. Mulliken, J. Am. Chem. Soc. 91 (1969) 4615.

[27] R.S. Berry, J. Chem. Phys. 45 (1966) 1228.

[28] C. Bordas, P.F. Brevet, M. Broyer, J. Chevaleyre, P. Labastie, J.P. Perrot. Phys. Rev. Lett. 60 (1988) 917.

[29] C. Bordas, P. Labastie, P.F. Brevet, M. Broyer. Phys. Rev. A 40 (1989) 1696.

[30] ZEKE Spectroscopy, E.W. Schlag (Cambridge University Press, 1998).

[31] M. Bixon and J. Jortner, J. Chem. Phys. 103 (1995) 4431.

[32] Modern Quantum Mechanics, J.J. Sakurai (The Benjamin/Cummings Pub. Co., Menlo Park, 1985).

[33] C. Bordas, F. Paulig, H. Helm, D.L. Huestis. Rev. of Sc. Instr. 67 (1996) 2257.

[34] B. Baguenard, J.B. Wills, F. Pagliarulo, F. Lépine, B. Climen, M. Barbaire, C. Clavier, M.A. Lebeault, C. Bordas. Rev. Sc. Instr. 75 (2004) 324-328.

[35] D. Ding, R.N. Compton, R.E. Haufler, C.E. Klots, J. Phys. Chem. 97 (1993) 2500.

[36] E.E.B. Campbell, K. Hansen, K. Hoffmann, G. Korn, M. Tchaplyguine, M. Wittmann, I.V. Hertel. Phys. Rev. Letters 84 (2000) 2128.

[37] H. Hohmann, R. Ehlich, S. Furrer, O. Kittelmann, J. Ringling, E.E.B. Campbell. Z. Phys. D 33 (1995) 143.

[38] B. Baguenard, J.C. Pinaré, F. Lépine, C. Bordas, M. Broyer. Chem. Phys. Letters. 352 (2002) 147.

[39] J. Simons, J. Am. Chem. Soc. 103 (1981) 3971.

[40] F. Lépine, A.R. Allouche, B. Baguenard, C. Bordas, M. Aubert-Frécon. J. Phys. Chem. A 106 (2002) 7177-7183 


\section{Figure captions:}

Figure 1: Schematic illustration of the vibrational autoionization process in a diatomic molecule. According to the energy of the Rydberg state under consideration, the exchange of one or several vibrational quanta is required. This leads to complex interferences in the autoionization process. In a polyatomic molecule, or in a cluster, the picture of delayed ionization is similar except for the larger number of vibrational degrees of freedom involved, and the correspondingly extremely higher density-of-states.

Figure 2: Experimental spectra of $\mathrm{Na}_{2}$ Rydberg states recorded with the intermediate level (v'=2, $\mathrm{J}^{\prime}=6$ ) for two different integration time (upper spectrum $200 \mathrm{~ns}$, lower spectrum $10 \mathrm{~ns}$ ). The $\Delta \mathrm{v}=1$ and $\Delta \mathrm{v}=2$ autoionization regions are indicated. Below the direct ionization threshold $\left(\mathrm{v}^{+}=2\right)$ and the $\Delta \mathrm{v}=1$ ionization threshold $\left(\mathrm{v}^{+}=1\right)$ lifetimes are noticeably longer. The external electric field $(\mathrm{F}=1.5 \mathrm{~V} / \mathrm{cm})$ is responsible for a sensible lowering of the ionization thresholds.

Figure 3: Spectrally averaged autoionization lifetimes as a function of energy for various electric field values. Solid curve: MQDT calculation $(F=0)$. Dashed curve: exp. with $F=2 \mathrm{~V} / \mathrm{cm}$. Dotted curve: exp. with F=5 V/cm. Dashed-dotted curve: exp. with F=20 V/cm.

Figure 4: Autoionization decays of the $17 \mathrm{~d}^{-}(\mathrm{v}=4, \mathrm{~J}=6)$ state for different values of the external electric field $\mathrm{F}$. At $\mathrm{F}=2.4 \mathrm{~V} / \mathrm{cm}$ the rise time $(60 \mathrm{~ns})$ is considerably larger than the laser pulse duration (about $10 \mathrm{~ns}$ ) and reveals a complex internal dynamics.

Figure 5: Delayed ionization of $\mathrm{C}_{60}$. Photoelectron kinetic energy spectrum recorded at delay $\tau=1 \mu$ s after the laser excitation (black dots). A fitting procedure according to eq. 16 (grey line) is used to extract a microcanonical temperature $T_{d}^{\exp }=3100 \pm 100 \mathrm{~K}$. This temperature is in excellent agreement with the temperature calculated from the total decay rate $T_{d}^{\text {th }}=3180 \mathrm{~K}$. 
Figure 6: Log-scale plot of the calculated (vibrational) density-of-states (number of states per interval of $10^{-4} \mathrm{eV}$ ) for $\mathrm{C}_{6}^{-}$(left) and $\mathrm{C}_{18}^{-}$(right). The excess energy is set to $1 \mathrm{eV}$. When the size of the system increases, the DOS losses its discrete structure and converges to the classical approximation. In the case of $\mathrm{C}_{18}^{-}$the classical limit is also plotted (dashed line).

Figure 7: Delayed photodetachment of $\mathrm{C}_{18}^{-}$(right). Experimental photoelectron kinetic energy spectrum recorded at $\tau=90 \mathrm{~ns}$ after laser excitation (straight line). Theoretical curve (dashed line) allows temperature estimation via a fitting procedure. The fitted temperature $(T=1400 \pm 100 \mathrm{~K})$ is in excellent agreement with the prediction of the Weisskopf model $(\mathrm{T}=1500 \mathrm{~K})$. As an example of small size system, similar spectrum obtained for $\mathrm{C}_{6}^{-}$is shown (left). No structure is observed in that case (owing to the broad initial internal energy distribution), however the simple model presented in the text is not able to describe this result. 


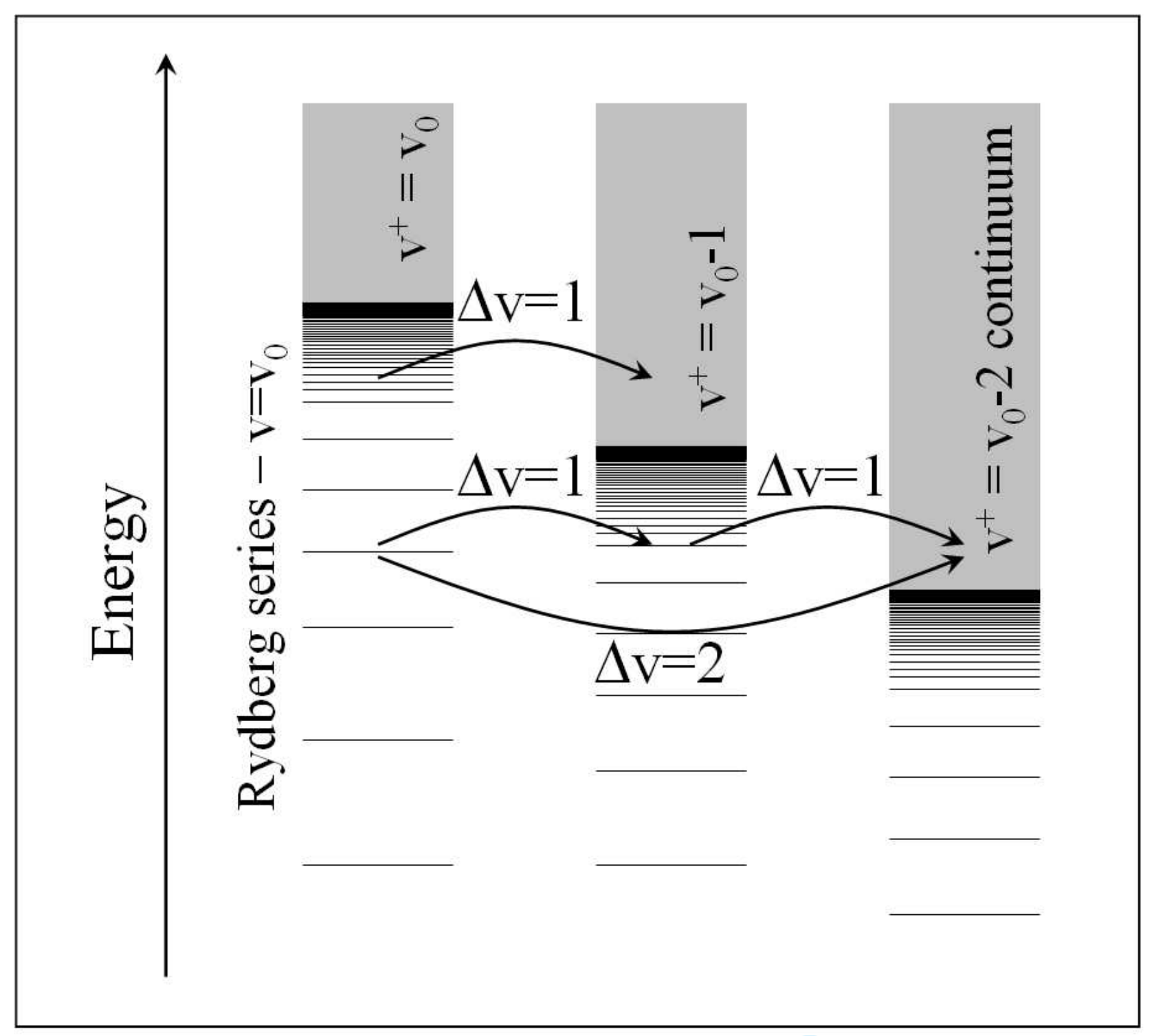

Figure 1 


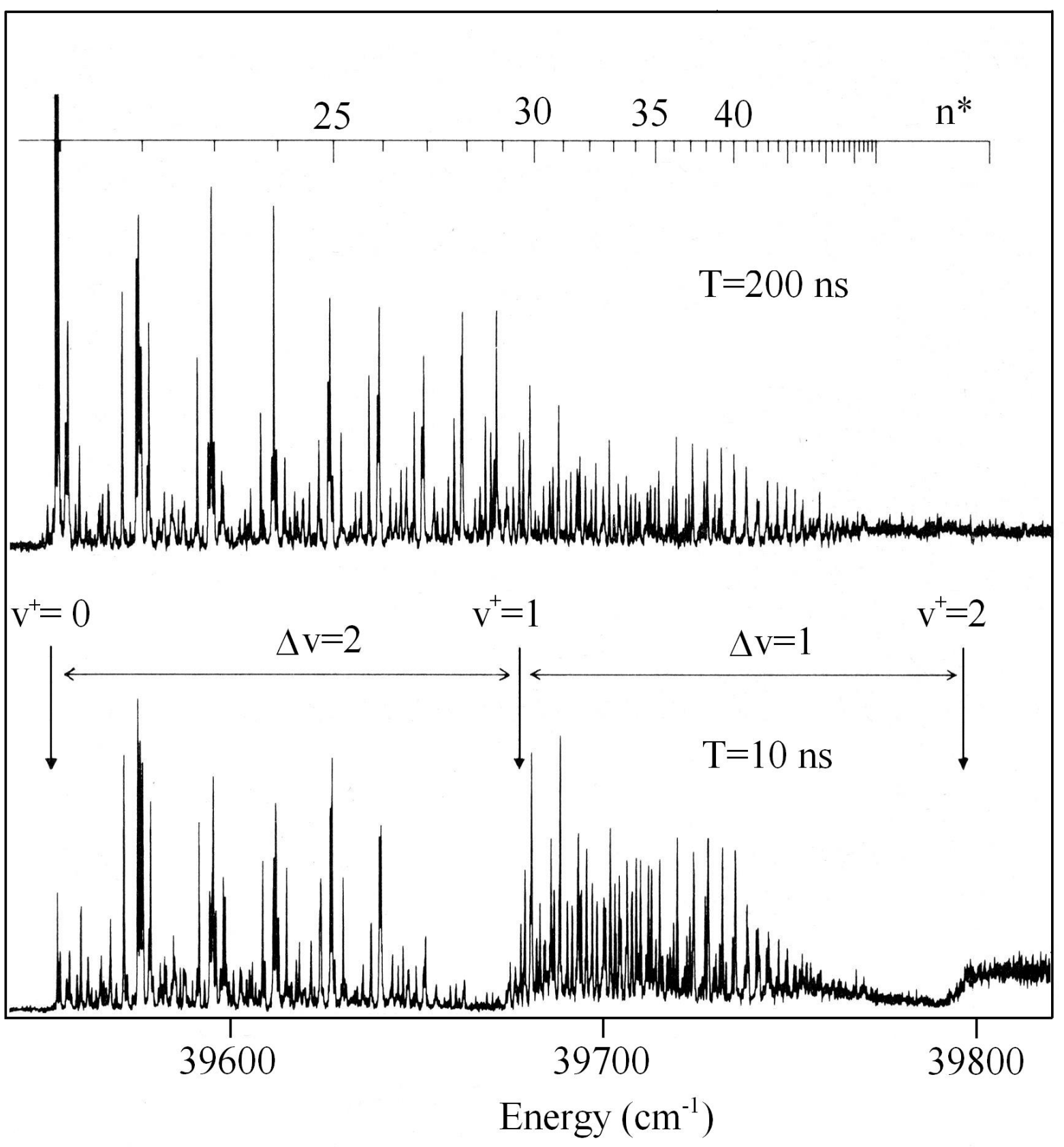

Figure 2 


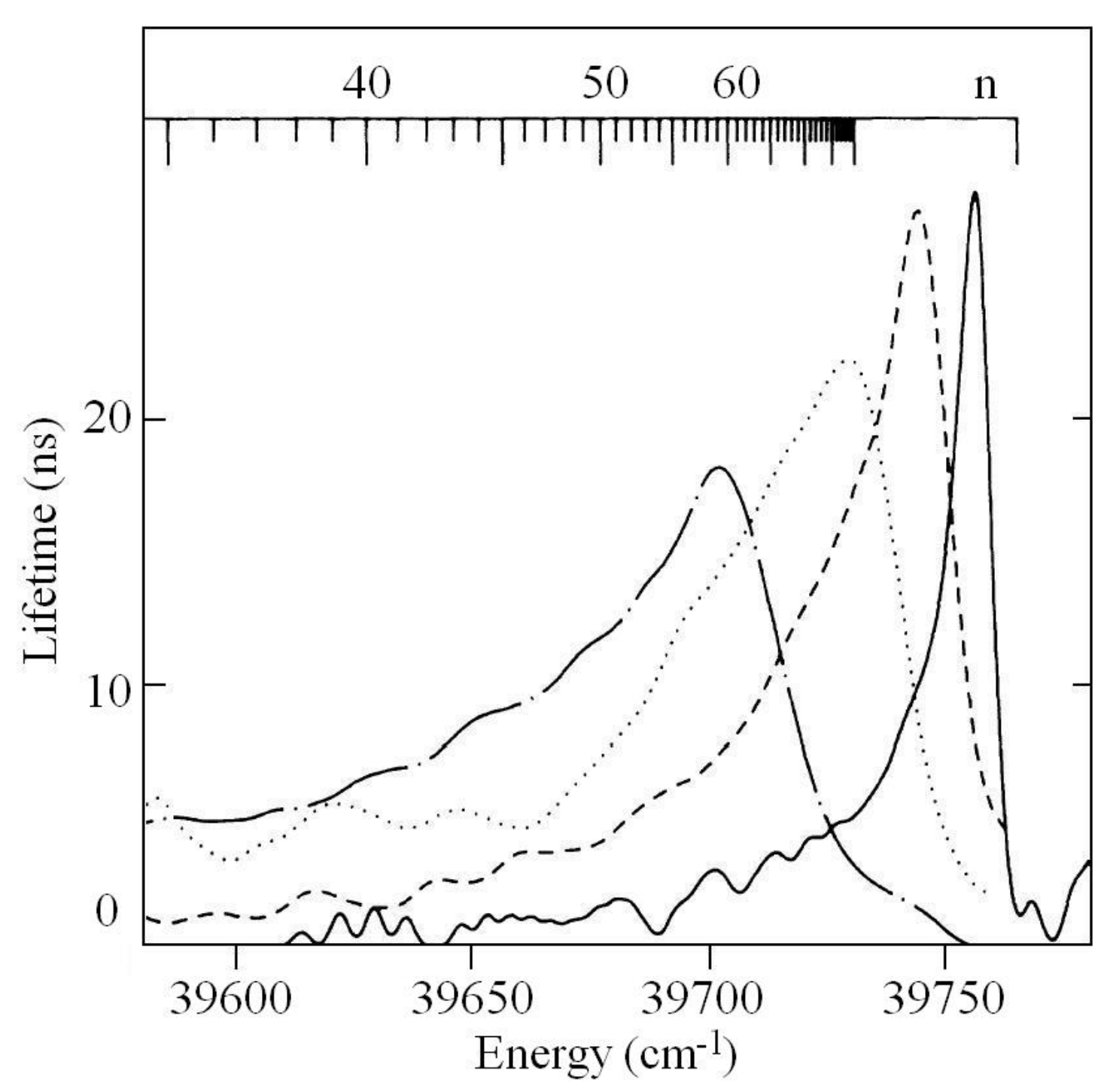

Figure 3 


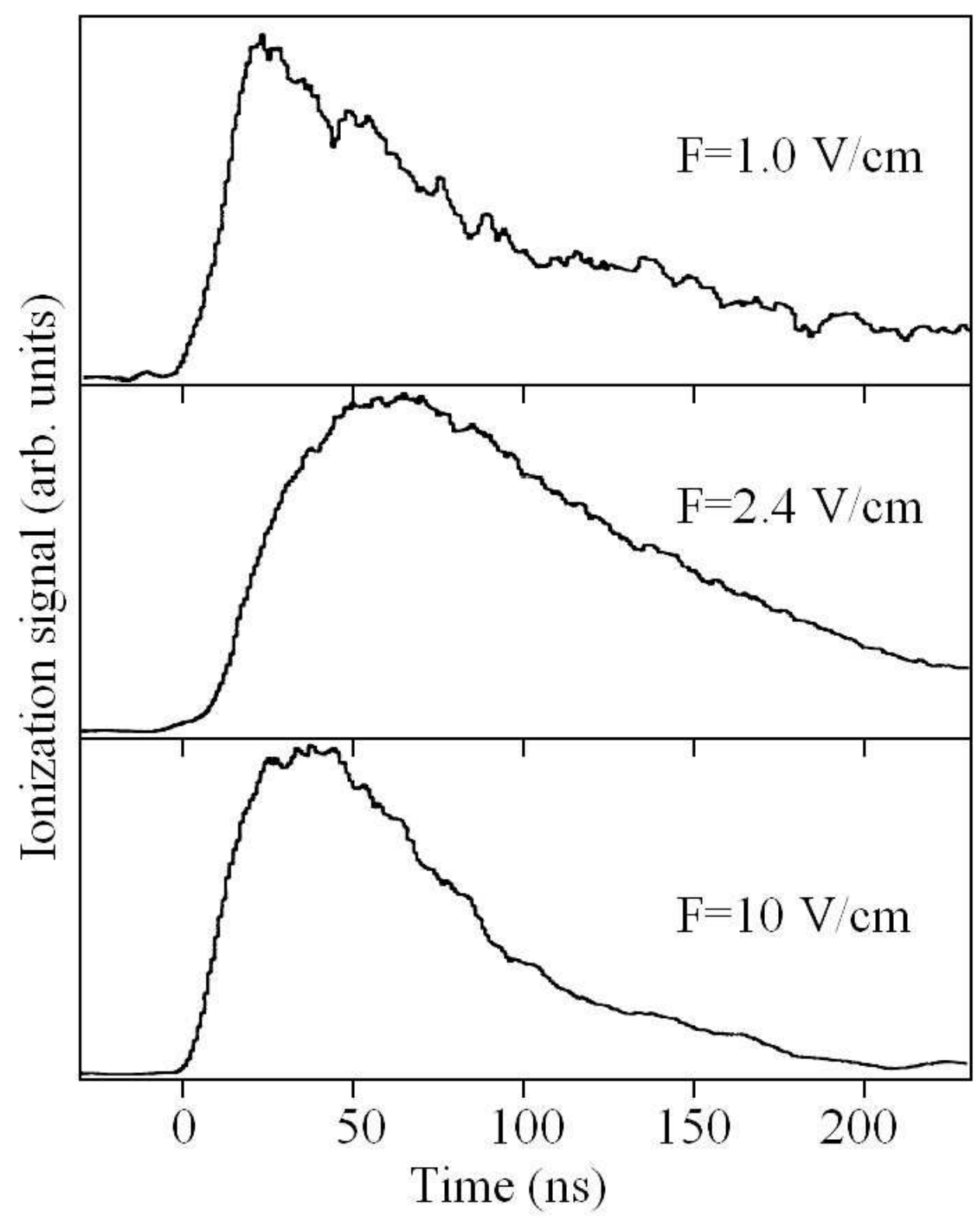

Figure 4 


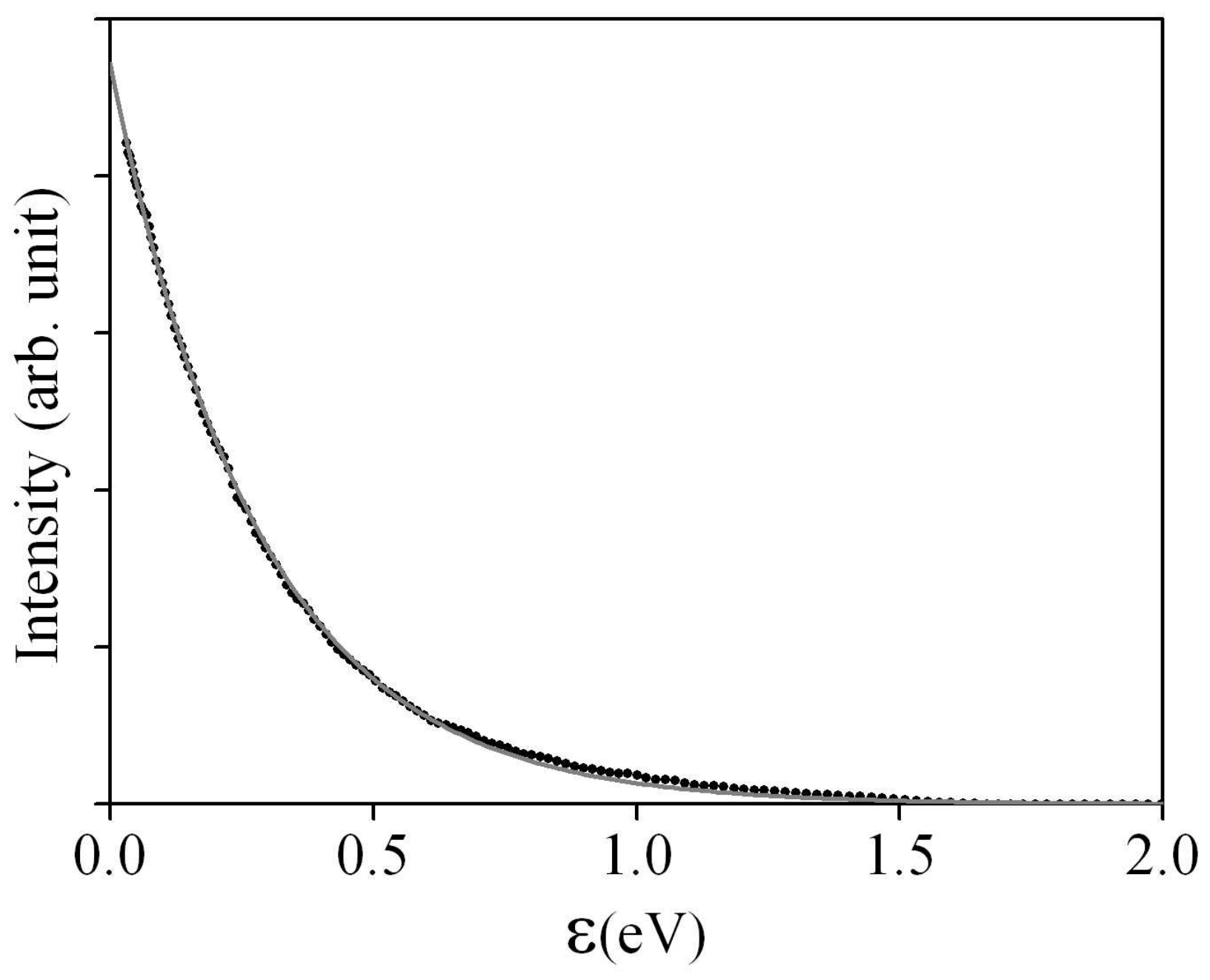

Figure 5 

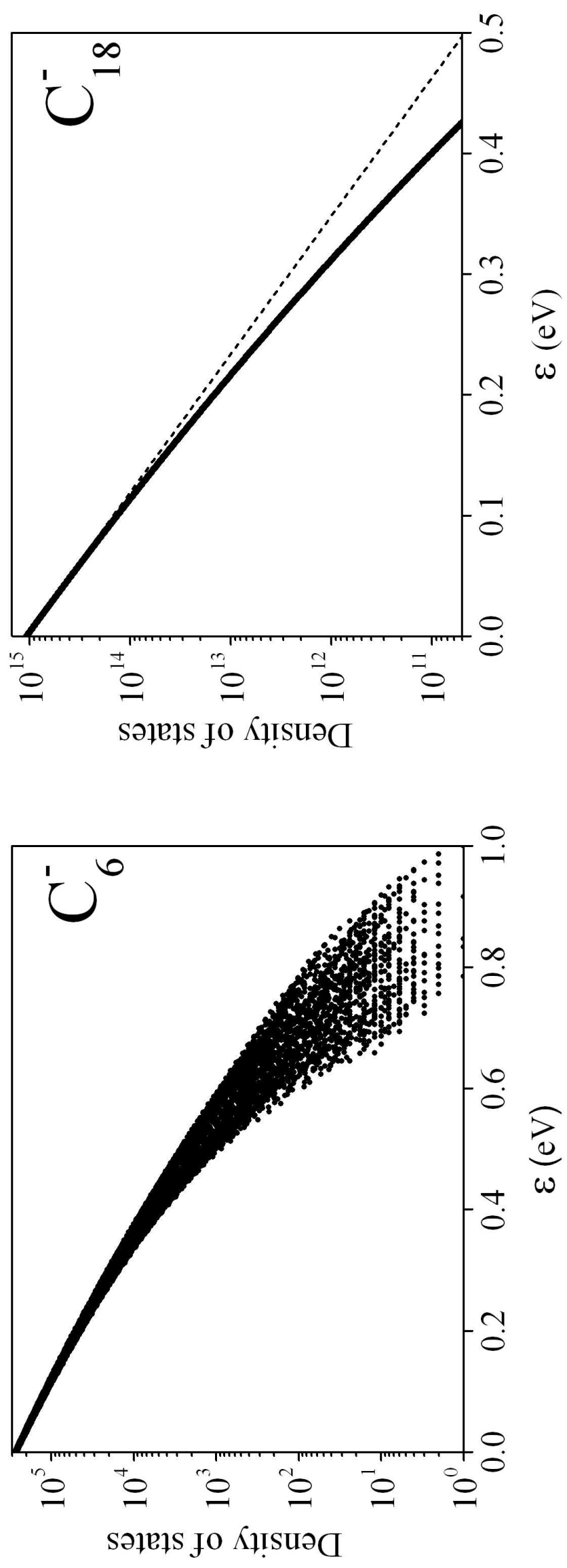

Figure 6 

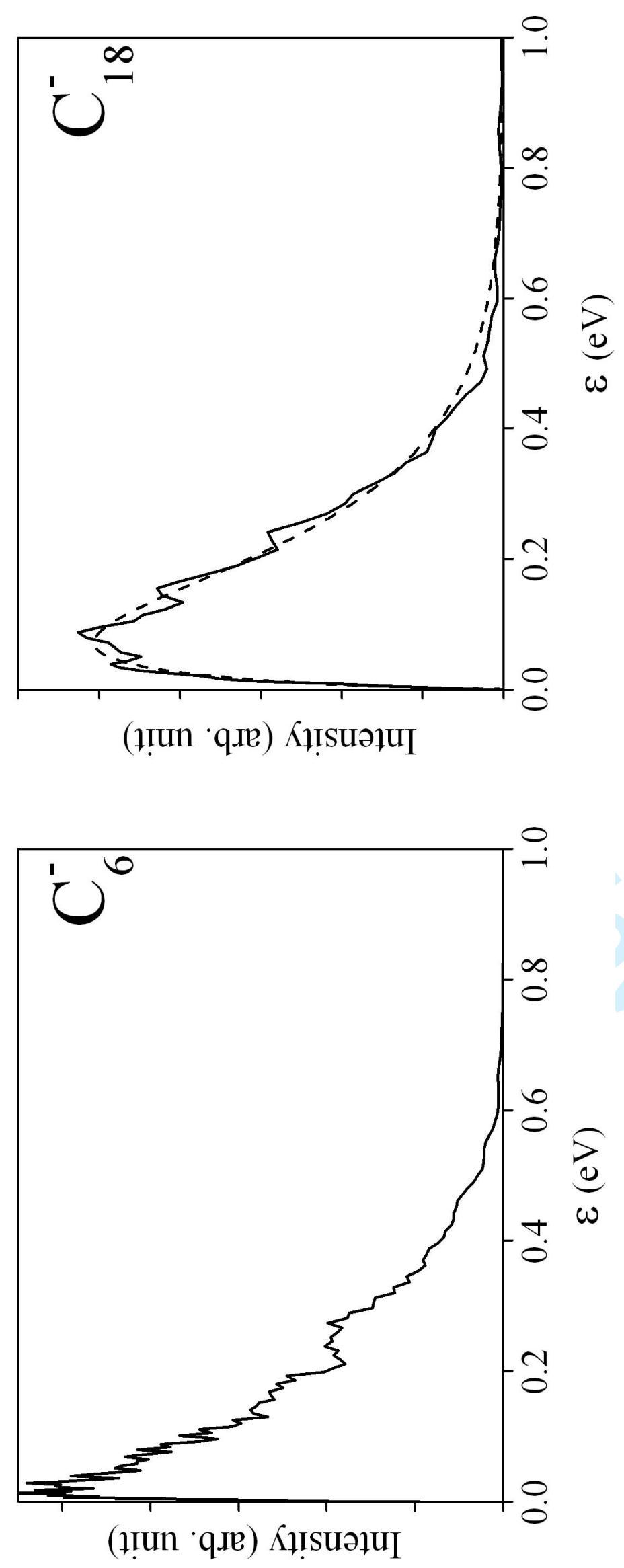

Figure 7 


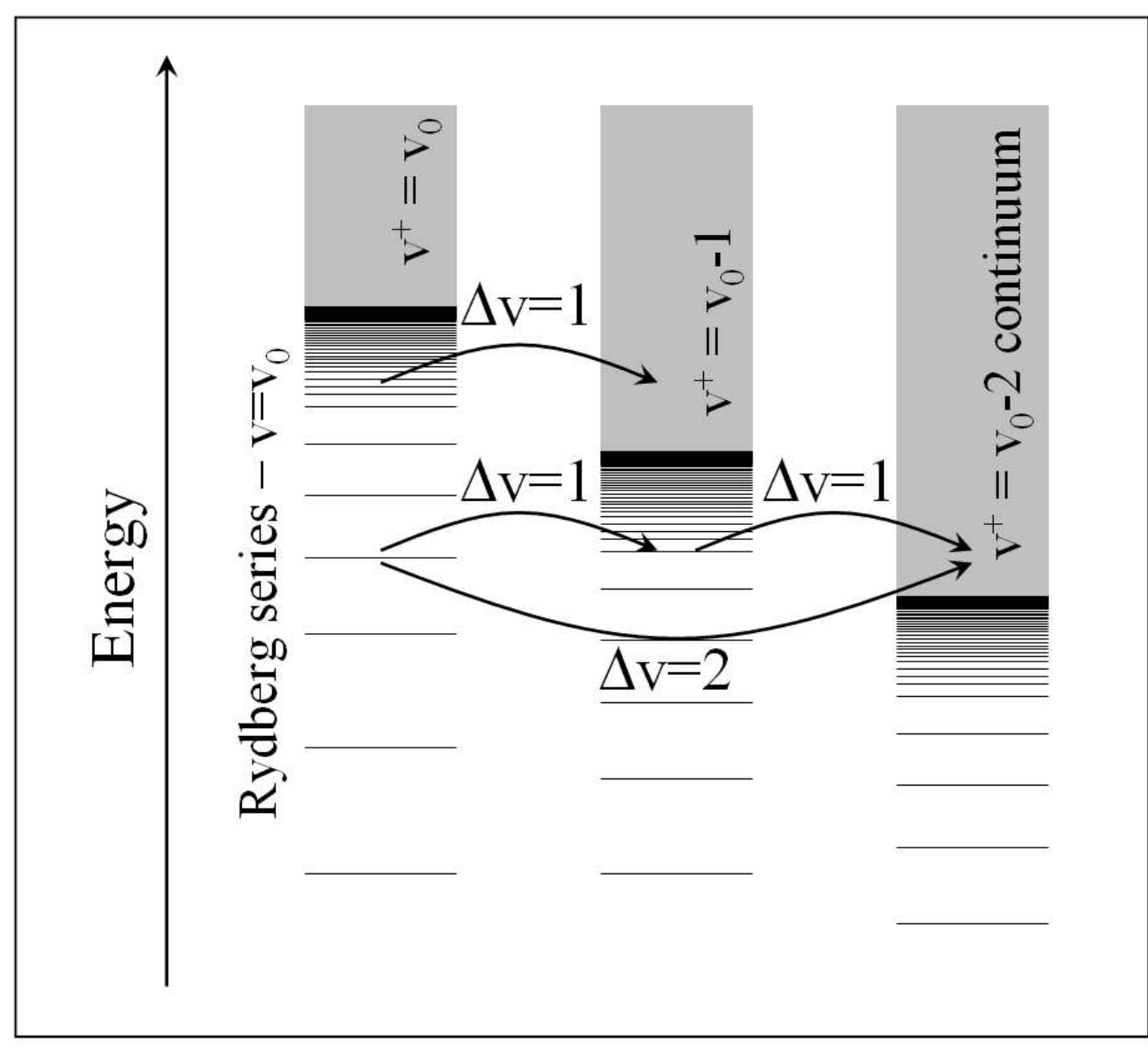

$234 \times 211 \mathrm{~mm}(100 \times 100 \mathrm{DPI})$

39

40

41

42

43

44

45

46

47

48

49

50

51

52

53

54

55

56

57

58

59

60

URL: http://mc.manuscriptcentral.com/tandf/tmph 


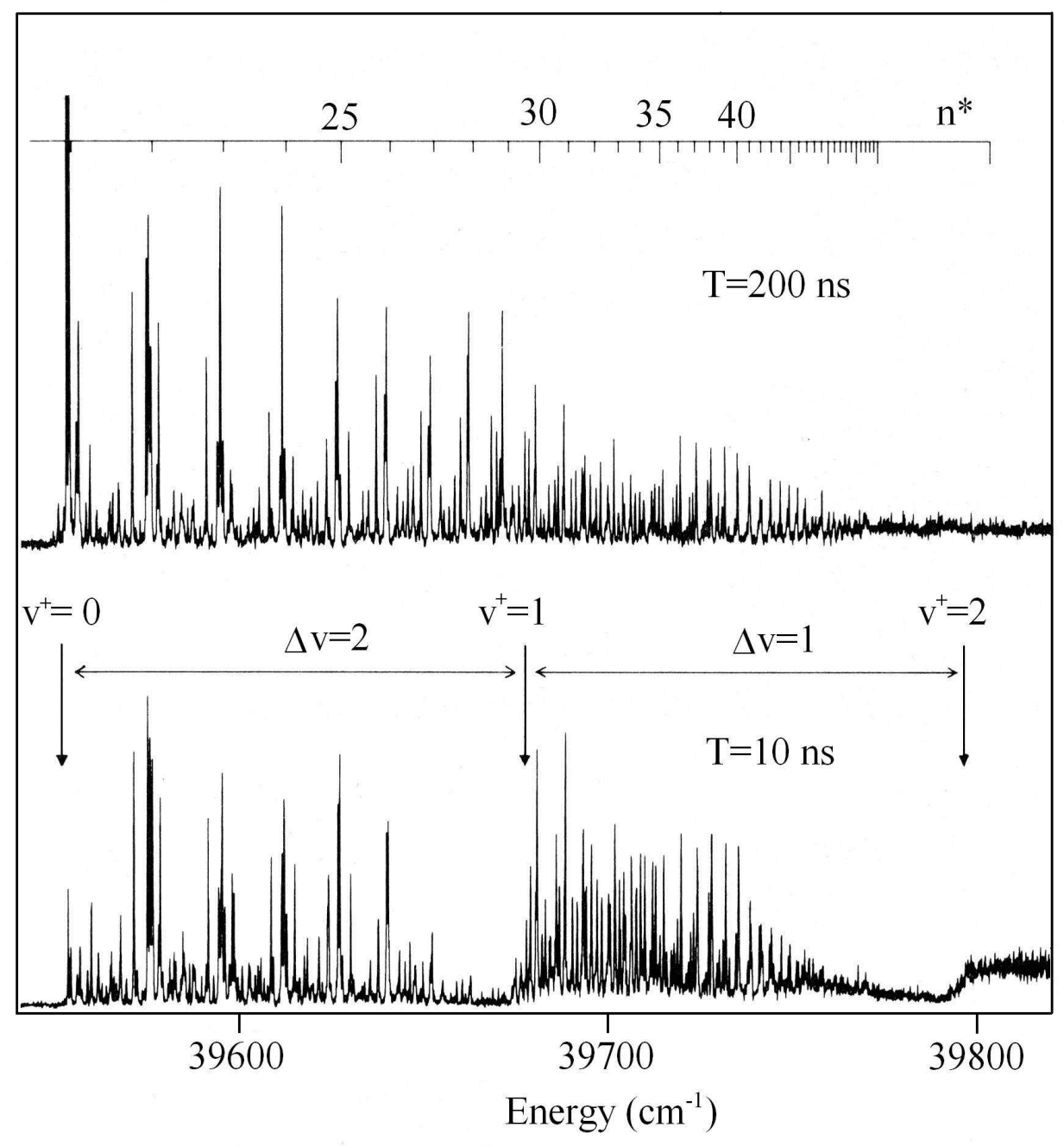

$486 \times 507 \mathrm{~mm}(96 \times 96 \mathrm{DPI})$ 


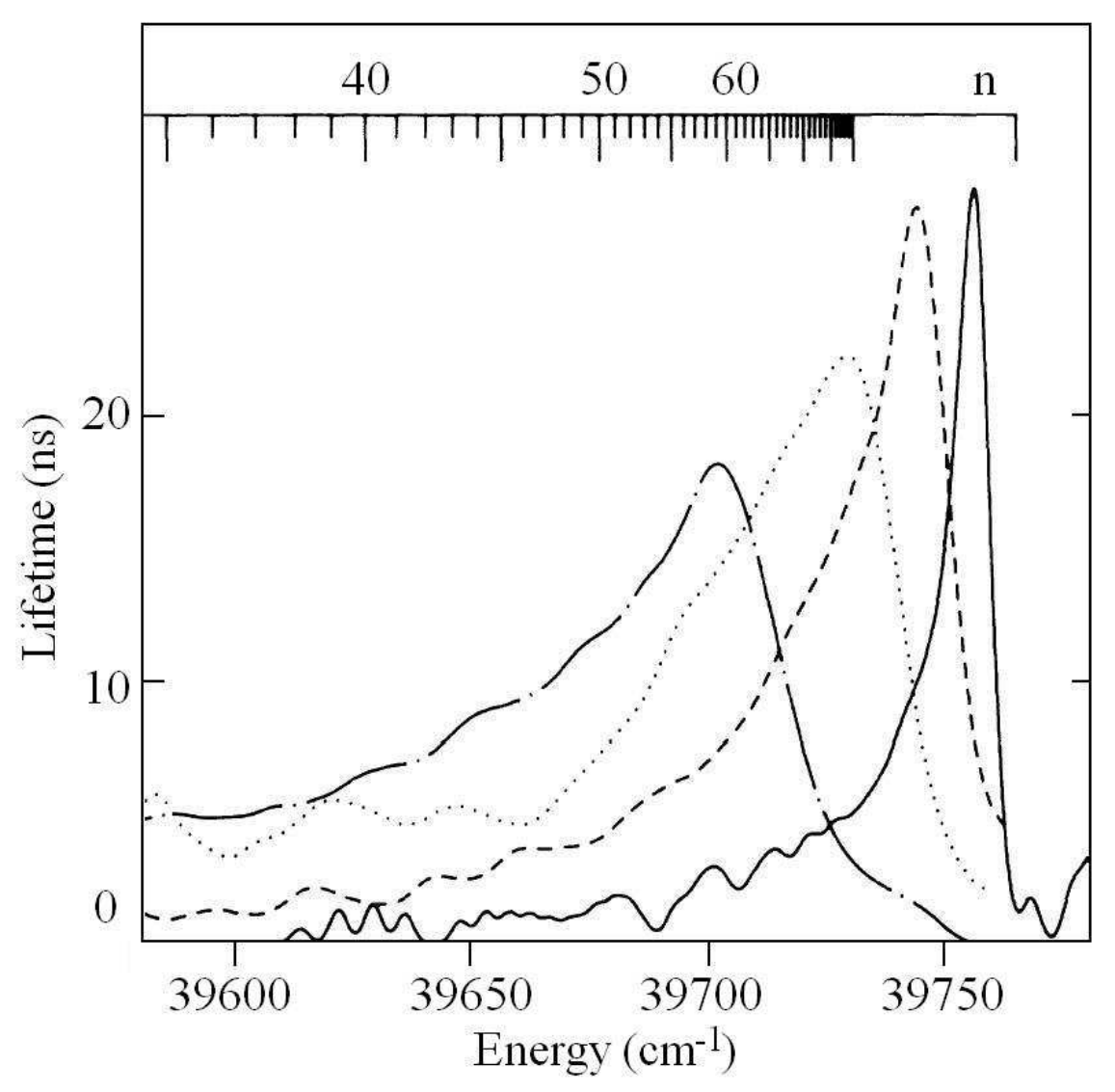

$254 \times 245 \mathrm{~mm}(96 \times 96 \mathrm{DPI})$

URL: http://mc.manuscriptcentral.com/tandf/tmph 


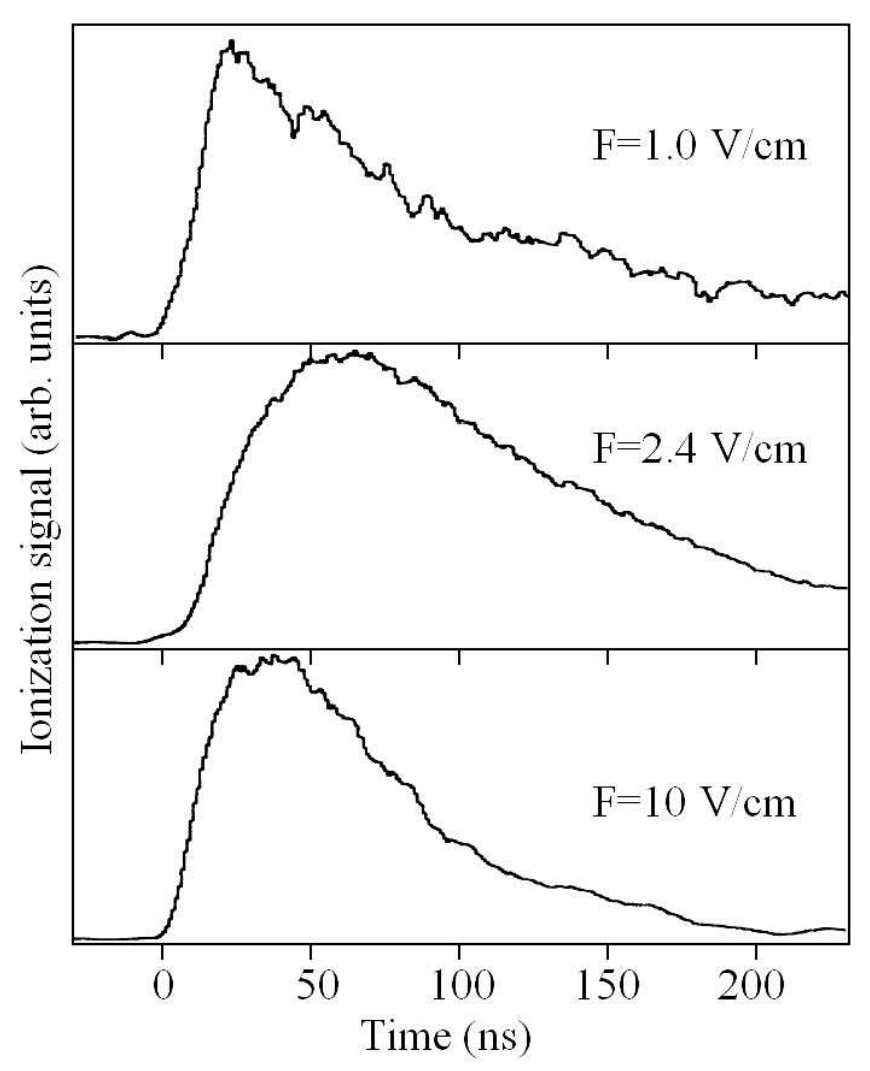

$286 \times 262 \mathrm{~mm}(96 \times 96 \mathrm{DPI})$

URL: http://mc.manuscriptcentral.com/tandf/tmph 


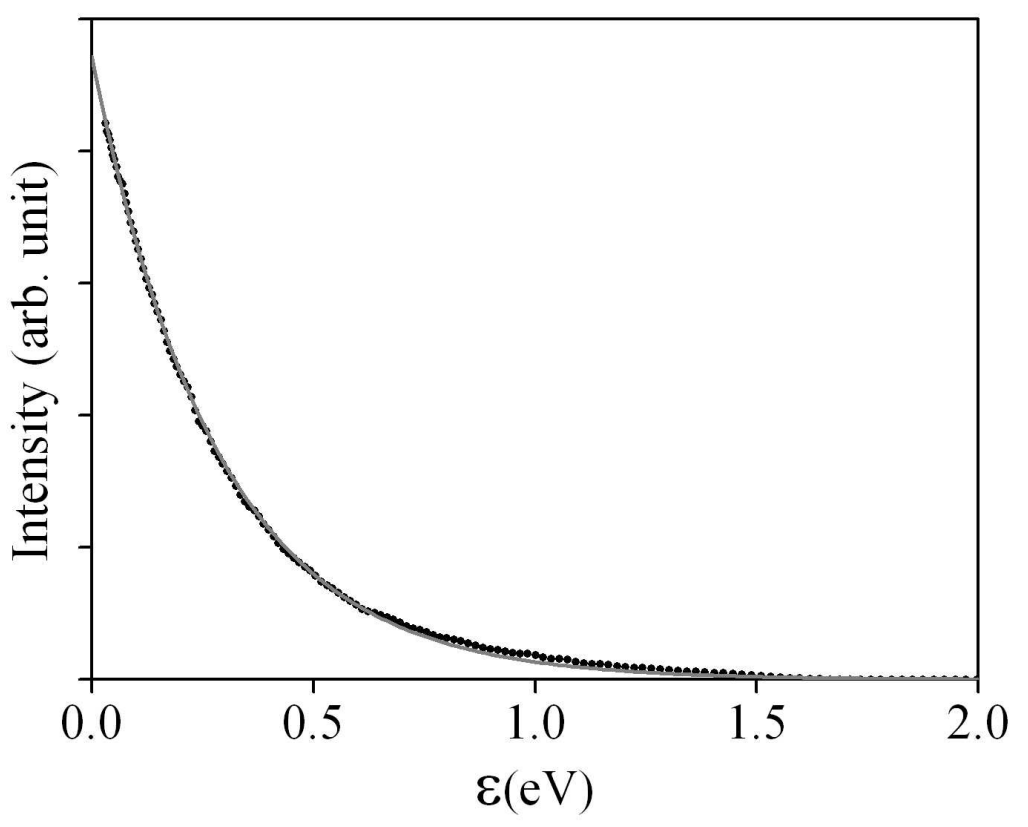

$297 \times 209 m m(150 \times 150$ DPI $)$

URL: http://mc.manuscriptcentral.com/tandf/tmph 


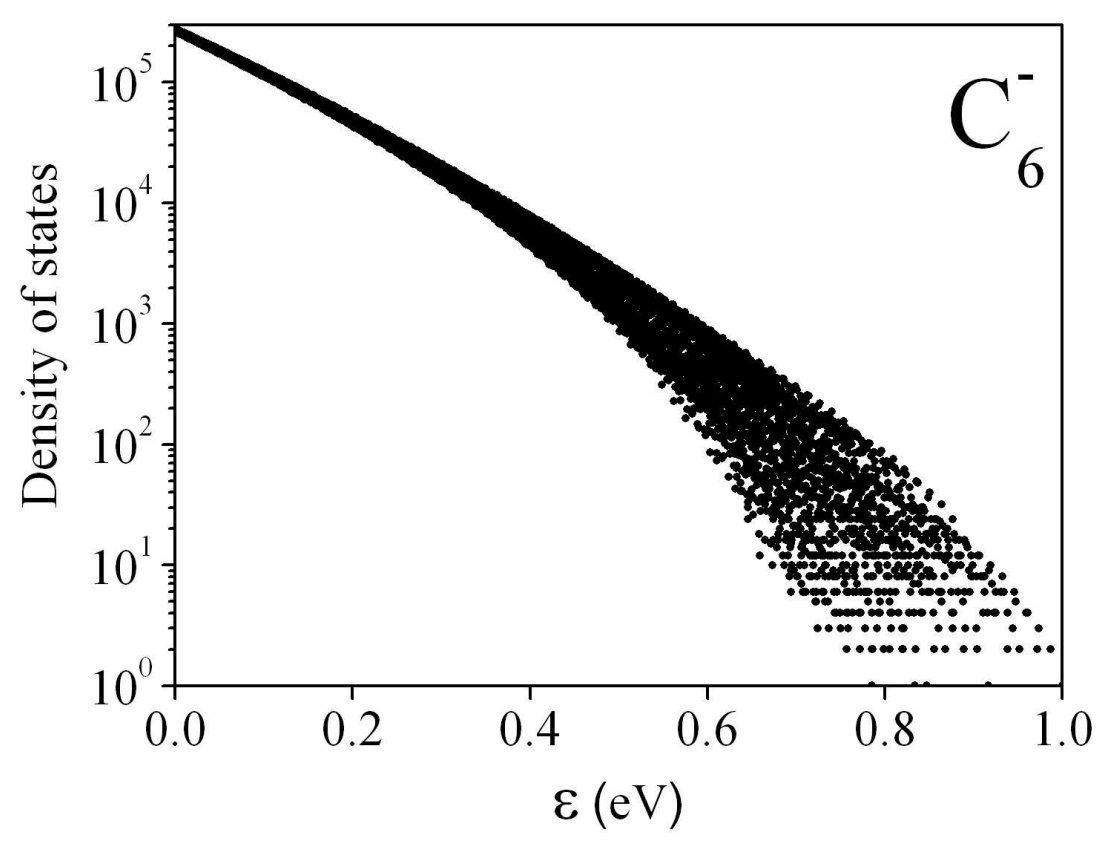

$297 \times 209 \mathrm{~mm}(150 \times 150 \mathrm{DPI})$ 


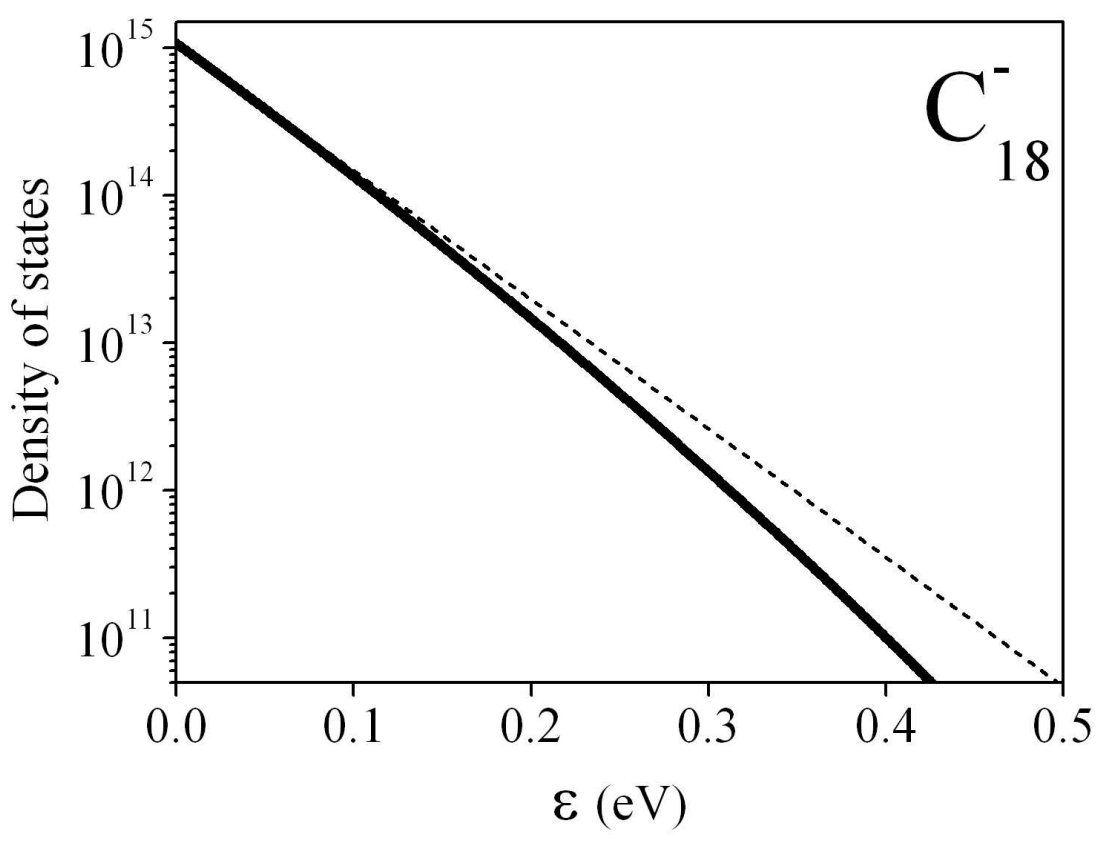

$297 \times 209 m m(150 \times 150$ DPI $)$ 


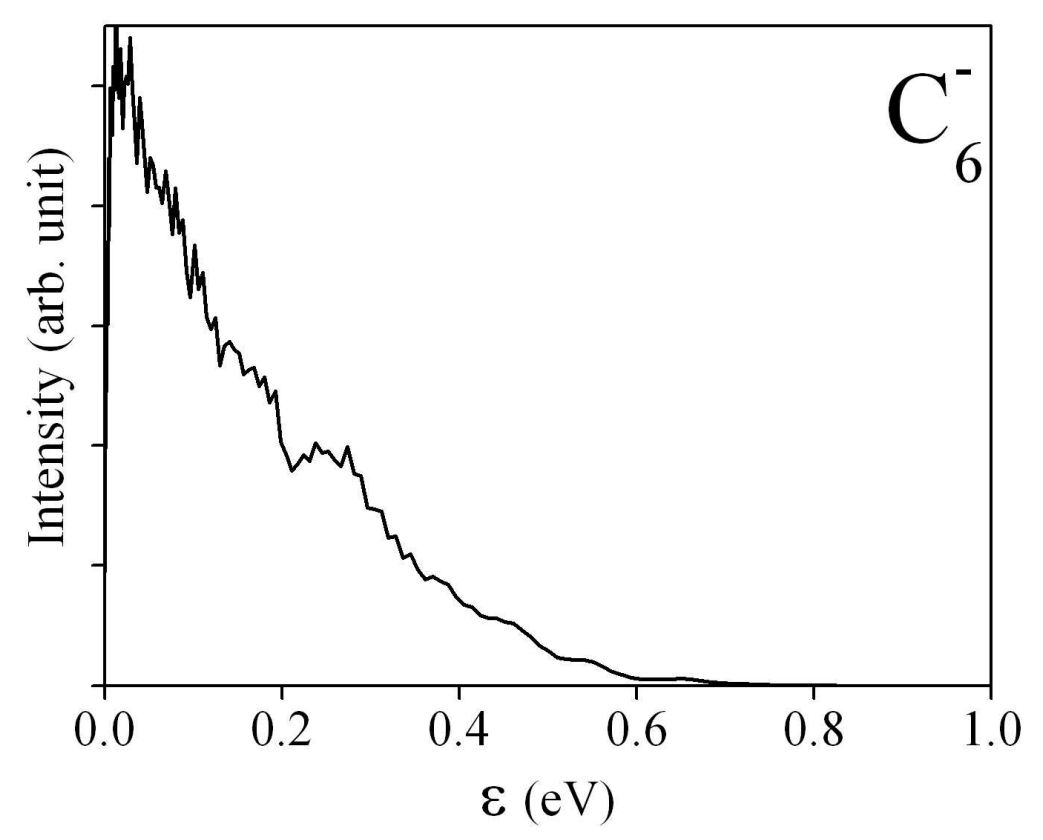

$297 \times 209 m m(150 \times 150$ DPI $)$ 


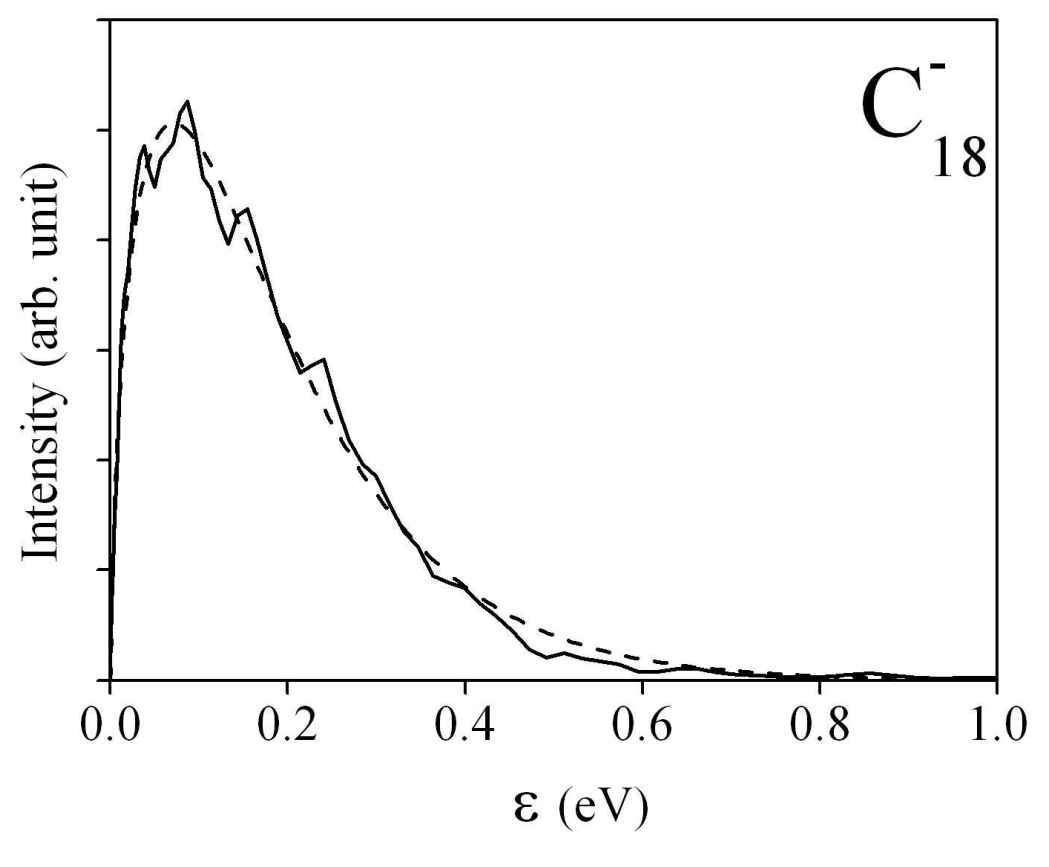

$297 \times 209 m m(150 \times 150$ DPI $)$ 